\title{
LA LOTTA ALLA CORRUZIONE IN AMBITO "REGIONALE": IL GRUPPO DI STATI CONTRO LA CORRUZIONE (GRECO)
}

\author{
Emanuele Vannata*
}

\author{
* University of Salerno (Italy), Department of Legal Sciences (School of Law) \\ (evannata@unisa.it)
}

\begin{abstract}
The article aims to retrace the role of the Council of Europe $(\mathrm{CoE})$ in the fight against corruption.

The analysis focuses on the institution of the Group of States against Corruption (GRECO), mandated to monitoring and evaluating the implementation of CoE instruments in subiecta materia, according to the standards contained in it, through the peer review evaluation mechanism.

A particular attention will be pledge to the corruption risks linked to the public health emergencies, in the light of the recent GRECO guidelines on preventing and managing corruption in the context of COVID-19 pandemic.
\end{abstract}

Keywords: Corruption, Council of Europe, COVID-19, GRECO. 


\title{
LUFTA KUNDËR KORRUPSIONIT NË NIVEL “RAJONAL”: GRUPI I SHTETEVE KUNDËR KORRUPSIONIT (GRECO)
}

\author{
Emanuele Vannata* \\ **Universiteti i Salernos, Departamenti i Shkencave Ligjore (Shkolla e së Drejtës) \\ (evannata@unisa.it)
}

\begin{abstract}
Abstrakt
Artikulli synon të rishikojë rolin e Këshillit të Europës (KiE) në luftën kundër korrupsionit.

Analiza fokusohet në institucionin e Grupit të Shteteve kundër Korrupsionit (GRECO), i mandatuar të monitorojë dhe vlerësojë zbatimin e instrumentave të KiE-së në këtë temë, sipas standardeve që përmbahen në të, nëpërmjet mekanizmit të vlerësimit të dyfishtë. Në vëmendje të veçantë do të jetë zotimi për rreziqet e korrupsionit lidhur me emergjencat e shëndetit publik, në linjë me udhëzimet e fundit të GRECO-s për parandalimin dhe menaxhimin e korrupsionit në kontekstin e pandemisë së COVID-19.
\end{abstract}

Fjalë kyçe: Korrupsioni, Këshilli i Europës, COVID-19, GRECO.

\section{Cenni introduttivi}

Il fenomeno corruttivo, nelle sue polimorfe manifestazioni, ha assunto da ben più di una decade una dimensione sovra-nazionale tale da giustificare l'elaborazione di tutta serie di strumenti giuridici con l'obiettivo di contrastare, in maniera più efficace e coordinata, $\mathrm{i}$ fenomeni corruttivi, nella loro ricaduta tanto interna quanto internazionale (1).

È ben noto, ormai, che la corruzione costituisca una seria minaccia al principio di legalità, alla democrazia e ai diritti umani, oltre che alla buona amministrazione, alla correttezza e all'uguaglianza sociale, senza considerare l'impatto significativo sullo sviluppo economico, sulla competizione e la crescita. Il costo economico e sociale della corruzione, pagato a caro prezzo dalla società civile, anche in termini di stabilità delle organizzazioni democratiche e di fiducia nelle pubbliche istituzioni, è figlio di una criminalità economica che trascende sempre più le geometrie statuali, cui le principali istituzioni sovra-nazionali hanno tentato di porre rimedio, a partire dagli anni Novanta del secolo scorso, attraverso strumenti tanto di c.d. soft law quanto di c.d. hard law.

Lo spazio giuridico europeo offre un apparato strumentale variegato, precipuamente indirizzato alla lotta alla corruzione e che, chiaramente, trova innanzitutto nell'Unione europea una sponda particolarmente felice (2), date le ormai ben note caratteristiche peculiari di tale Organizzazione, che fanno di essa "il fenomeno di regionalizzazione più 'riuscito' del sistema globale" (3).

Tuttavia, risulta al pari rilevante - nel quadro degli strumenti giuridici "regionali" specificamente dedicati alla corruzione (4) - il contributo del Consiglio d'Europa 
(Council of Europe/CoE) (5), la più risalente organizzazione intergovernativa europea, nata nel 1949 allo scopo di “attuare un'unione più stretta fra i Membri per tutelare e promuovere gli ideali e i principi che sono loro comune patrimonio e per favorire il loro progresso economico e sociale" (6), in particolare la triade diritti umani-democrazia-stato di diritto.

All'ampio impegno internazionale nel contrasto a tale fenomenologia criminosa, in termini di risposta normativa, vuoi sul piano universale che regionale, si accompagna una non sempre uniforme scelta dell'approccio impiegato, spesso altalenante tra logiche meramente repressive e caratteri più marcatamente preventivi (7).

In tale ampio contesto, si colloca l'attività del Consiglio d'Europa, improntata anzitutto ad una certa dinamicità e multidisciplinarietà dell'approccio strategico (8), a partire dall'istituzione del Gruppo Multidisciplinare sulla Corruzione (GMC) nel 1994 e dall'adozione - nel 1996 - del Programma d'Azione contro la Corruzione (9), che costituisce essenzialmente l'architrave su cui poggiano le attività del Consiglio e la loro successiva articolazione. Accanto alla promozione e alla stipula di accordi o convenzioni tra Stati membri (ma a cui sovente partecipano anche Stati terzi), improntate ad un'ottica di complementarietà, quali la Convenzione penale sulla corruzione del 1999 (10) (e il successivo Protocollo addizionale del 2003 (11)) e la Convenzione civile sulla corruzione (12) del medesimo anno, il Consiglio ha ritenuto anche di affiancare un organo di controllo - il Gruppo di Stati contro la Corruzione (GRECO) (13) - al fine di monitorare e valutare il rispetto degli obblighi giuridici da essi derivanti (14).

Il presente lavoro mira, infatti, a ripercorrere il contributo del $\mathrm{CoE}$ nella lotta alla corruzione in ambito "regionale", a partire innanzitutto dall'istituzione del GRECO e dal suo funzionamento, per poi soffermarsi sui rischi corruttivi nel contesto dell'emergenza sanitaria causata dalla pandemia di COVID-19 e sull'apporto del Gruppo alla prevenzione della corruzione attraverso l'emanazione delle guidelines del 15 aprile 2020 (15).

\section{La strategia anti-corruption del Consiglio d'Europa}

La dimensione "pan-europea" del Consiglio d'Europa (16), che le consente di operare in uno spazio giuridico che ingloba tutti gli Stati membri dell'Unione europea e che conta attualmente 47 Stati membri, è senz'altro un aspetto da salutare con favore. Infatti, se si considera, da un lato la già evidenziata e crescente transnazionalità del fenomeno corruttivo e, dall'altro, la questione culturale sottesa alle pratiche corruttive - che mettono a repentaglio alcuni dei principi ordinatori della stabilità del sistema interstatuale e delle comunità nazionali (17) - allora appare quasi evidente che ritrovarsi dinanzi ad una "geografia che trascende quella dei geografi" (18) può contribuire al buon esito delle azioni (comuni) contro la corruzione nell'area europea.

Sulla scorta di quei valori spirituali e morali condivisi da tutti gli Stati membri "che sono patrimonio comune dei loro popoli e fondamento dei principi di libertà personale, libertà politica e preminenza del Diritto, dai quali dipende ogni vera democrazia" (19), inevitabilmente a rischio compromissione dalle condotte corruttive, il $\mathrm{CoE}$ ha assunto un ruolo di primo piano nella lotta alla corruzione. A partire dal settembre del 1994 (20), infatti, raccogliendo l'input proveniente dai Ministri europei riuniti alla Conferenza di La Valletta (21) di "set up a multi-disciplinary Group on Corruption within the Council of Europe, under the responsibility of the European Committee on Crime Problems (CDPC) and the European Committee on Legal Co-operation (CDCJ), and entrust the Group with 
the task of examining what measures might be suitable to be included in a programme of action at international level against corruption" (22), il Comitato dei Ministri del CoE istituì il Gruppo Multidisciplinare sulla Corruzione (Multidisciplinary Group on Corruption - GMG), il quale predispose il Programma d'Azione contro la Corruzione del 1995, che venne definitivamente adottato nel novembre del 1996. Tale Programma, da cui già emergeva la consapevolezza del fenomeno "as a problem of society" (23) e rispetto cui "no system of government and administration is immune (...) by those intent on the abuse of power" (24), risulta essere il punto di svolta per l'acquisizione del decisivo impulso politico (ma anche normativo) atto a traghettare la lotta alla corruzione tra gli obiettivi prioritari ed essenziali del CoE. Da un punto di vista normativo, infatti, il Programma si pone esattamente all'origine (e a fondamento) dei sei strumenti adottati negli anni a seguire e che costituiscono l'ossatura della strategia anti-corruption del CoE. A distanza di soltanto un anno dalla definitiva adozione del Programma, seguì, difatti, l'emanazione della Risoluzione "sui Venti Principi Guida per la Lotta alla Corruzione" del 1997 (25), tra i quali "to ensure co-ordinated criminalisation of national and international corruption" (26), "to promote the specialisation of persons or bodies in charge of fighting corruption and to provide them with appropriate means and training to perform their tasks" (27), "to ensure that the organisation, functioning and decisionmaking processes of public administrations take into account the need to combat corruption" (28), nonchè "to develop to the widest extent possible international cooperation in all areas of the fight against corruption" (29). Tali principi si inquadrano in una prospettiva general-preventiva e, allo stesso tempo, aspirano alla promozione di good practices etiche, prendendo in particolare considerazione la libertà di informazione, la trasparenza dei meccanismi decisionali, il controllo dei conti, i codici di buona condotta dei funzionari, il finanziamento dei partiti e delle campagne elettorali (30), con un marcato valore politico e di indirizzo, pur nella consapevolezza della sua del tutto esigua portata perentoria.

Permanendo in tale ambito, in un quadro di più marcata soft law, si sono poi susseguite, nel nuovo millennio, la Raccomandazione sui Codici di Condotta dei Pubblici Ufficiali del 2000 (31) e la Raccomandazione sulle Regole Comuni contro la Corruzione nel Finanziamento ai Partiti Politici e alle Campagne Elettorali del 2003 (32).

La prima è essenzialmente volta a raccomandare agli Stati membri di promuovere l'adozione di codici di condotta nazionali per i dipendenti pubblici sulla base del modello delineato dal Model Code annesso alla Raccomandazione, nella prospettiva di definire le regole comportamentali dei dipendenti delle amministrazioni pubbliche, costruendo una cornice deontologica entro la quale esercitare il servizio pubblico (33). La seconda, invece, detta le regole comuni, riprodotte nell'Appendice alla Raccomandazione, destinate ad orientare i governi degli Stati membri nell'adozione, nei loro rispettivi ordinamenti, di norme in materia di finanziamento pubblico dei partiti e delle campagne elettorali.

Tuttavia, è ben noto che buona parte dell'attività del $\mathrm{CoE}$ è teleologicamente finalizzata alla conclusione di accordi o convenzioni tra gli Stati membri (ma aperte anche a Stati terzi). Difatti, gli sforzi del CoE si sono tradotti soprattutto nell'adozione delle due convenzioni sulla corruzione, in materia penale ed in materia civile, ambedue aperte alla firma nel 1999, nonché del Protocollo addizionale alla Convenzione penale del 2003.

In particolare, la Convenzione penale sulla corruzione del 1999, anticipata, soltanto di un paio di anni, dalla Convenzione dell’Organizzazione per la Cooperazione e lo Sviluppo 
Economico (OCSE) sulla lotta alla corruzione di pubblici ufficiali stranieri nelle operazioni economiche internazionali del 1997 (34), rappresenta senz'altro uno dei primissimi strumenti multilaterali posti in essere nella materia in esame a livello internazionale. La sua portata, alquanto vasta, copre entrambe le forme di corruzione attiva e passiva (35), rispetto ad una vasta gamma di attori (e.g., pubblici ufficiali nazionali e stranieri, membri dei parlamenti nazionali e stranieri, membri di assemblee parlamentari internazionali, funzionari internazionali, giudici nazionali, stranieri ed internazionali), nonché le forme di comportamento corrotto considerate normalmente come tipi specifici di corruzione, quali il traffico di influenze attivo e passivo, il riciclaggio dei proventi della corruzione, i reati contabili connessi con reati di corruzione. La Convenzione, inoltre, prende in considerazione anche la corruzione nel settore privato, richiedendo agli Stati di prevedere sanzioni e misure efficaci e dissuasive, anche nei confronti delle persone giuridiche, le quali dovranno essere, anch'esse, penalmente responsabili per i reati commessi a proprio vantaggio e destinatarie di efficaci sanzioni penali o civili. Invece, il Protocollo addizionale è intervenuto a distanza di sei anni dalla sua Convenzione "madre" a scopo estensivo, ampliandone sostanzialmente la portata agli arbitri in questioni commerciali, civili e di altra natura e ai giurati.

La prospettiva di affrontare il fenomeno corruttivo in maniera "globale" ha condotto gli Stati membri del CoE e gli altri Stati firmatari, "convinced of the importance for civil law to contribute to the fight against corruption, in particular by enabling persons who have suffered damage to receive fair compensation" (36), a pervenire all'adozione di uno strumento giuridico convenzionale in grado di definire norme comuni internazionali anche con gli strumenti propri del diritto civile (ad esempio, in termini di risarcimento del danno, responsabilità civile, validità dei contratti, whistleblowing (37), formazione della prova, misure cautelari), obbligando le Parti Contraenti a prevedere nelle loro legislazioni nazionali "rimedi giudiziali efficaci a favore delle persone che hanno subito un danno risultante da un atto di corruzione, al fine di consentire alle medesime di tutelare i propri diritti ed interessi, ivi compresa la possibilità di ottenere il risarcimento dei danni" (38).

\subsection{Il GRECO}

Affianco alle sue fonti legali, senz'altro volte a migliorare la capacità degli Stati di incidere sul fenomeno corruttivo sia a livello nazionale che internazionale, il CoE, come già anticipato, si è dotato di un organo di controllo (39), destinato "to improve the capacity of its members to fight corruption by following up, through a dynamic process of mutual evaluation and peer pressure, [in] compliance with their undertakings in this field" (40). Già a partire dal 1997, i Capi di Stato e di Governo degli Stati membri del CoE, in occasione del secondo Summit di Strasburgo (41), avevano sollecitato il Comitato dei Ministri "to establish without delay an appropriate and efficient mechanism for monitoring observance of the guiding principles and implementation of the said international legal instruments" (42). Tale indirizzo era stato poi accolto dal Comitato nel novembre 1997, durante la sua 101esima Sessione, con la Risoluzione 97/24, attraverso la quale i Ministri affidarono al GMC il mandato a definire, al più presto, un draft text finalizzato all'istituzione del meccanismo richiesto. Nel marzo del 1998 è in seguito intervenuta l'approvazione del Draft Agreement da parte del GMC, a cui è seguita la Risoluzione 98/7 del Comitato che ha autorizzato l'istituzione del GRECO (43). La 
definitiva istituzione dell'organo è intervenuta nel maggio 1999 con la Risoluzione 99/5 (44).

Tale organismo, con sede a Strasburgo, si pone il ragguardevole obiettivo di vigilare sull'osservanza dei Principi Guida per la lotta alla corruzione, nonché di monitorare l'implementazione di tutti gli strumenti giuridici testé evidenziati ed è stato dotato di uno Statuto (45), che ne disciplina l'organizzazione ed il funzionamento, a cui si accompagnano le Regole di procedura adottate dall'organo medesimo in seno alla sua prima Sessione plenaria (46).

L'adesione al GRECO è aperta a tutti gli Stati, ancorché non membri del CoE, ma che hanno partecipato all'Enlarged Partial Agreement, ovvero a Stati che aderiscono agli strumenti internazionali adottati dal CoE (47). Inoltre, è anche conferito al Comitato dei Ministri del CoE, nella sua composizione ristretta agli Stati membri dell' Enlarged Partial Agreement, il potere di invitare all'adesione uno Stato non membro, anche se non rientrante tra le ipotesi poc'anzi citate (48). Lo Stato aderente, a seguito dell'invito, è però tenuto ad accettare, con dichiarazione separata, l'applicazione dei Principi Guida (49). È bene considerare anche che ogni Stato che ratifichi tanto la Convenzione penale quanto quella civile sulla corruzione diviene automaticamente membro del GRECO e accetta le sue procedure di valutazione (50).

Attualmente, il Gruppo comprende 50 Stati membri, di cui 48 di area geografica europea e 2 extra-europea (Kazakhstan e Stati Uniti). Gli Stati operano nel GRECO attraverso la propria delegazione, composta da due rappresentanti da essi nominati (51), con diritto di voto nell'Assemblea plenaria, ma forniscono anche una lista di cinque esperti disponibili a prendere parte ai procedimenti di valutazione (52). La struttura del GRECO si articola, inoltre, in un Bureau (53), composto dal Presidente e dal Vicepresidente e da altre cinque persone elette dal Gruppo tra i rappresentanti degli Stati "entitled to vote" (54), e in una Statutory Committee (55), in cui siedono i rappresentanti degli Stati membri del CoE nel Comitato dei Ministri che sono anche membri del GRECO e i rappresentanti all'uopo designati dagli altri membri del Gruppo. A chiudere, infine, il quadro strutturale è il Secretariat, che ha funzioni di assistenza e supporto amministrativo del GRECO (56).

Al netto dell'apparato organigrammatico, appare senz'altro di indubbio interesse il funzionamento del sistema sotteso ai processi valutativi e di controllo che stanno alla base della mission del Gruppo. Anzitutto, secondo quanto stabilito dallo Statuto, il procedimento di valutazione opera in cicli (Evaluation Round) (57), ognuno relativo ad uno o più temi specifici. Sovrintende al procedimento il principio della mutua valutazione o valutazione reciproca (mutual evaluation) e di mutua pressione (peer pressure), a cui si ispirano il monitoraggio e la valutazione (58), composto di due parti: una valutazione reciproca e una procedura di conformità, indistintamente applicati a tutti gli stati membri in ogni Round di Valutazione.

Il meccanismo della peer review rappresenta, difatti, un processo dinamico di valutazione, condotto da pari (ovvero gli esperti degli stessi Stati membri) sulla base degli elementi raccolti dalla compilazione di questionari, dai colloqui effettuati nel corso delle visite (onsite visits), oltre che dai commenti e dalle osservazioni forniti dallo Stato valutato. Alla base della valutazione reciproca vi è, infatti, la raccolta di informazioni mediante la compilazione di un Questionnaire, adottato dal GRECO per ogni Round e sottoposto a tutti gli Stati membri, i quali sono tenuti a fornire le risposte al Segretariato entro i termini fissati dal Gruppo (59). È proprio in questa fase della procedura che il GRECO ha la possibilità di inviare in loco un'équipe di esperti "for the purpose of seeking additional 
information concerning its law or practice, which is useful for the evaluation" (60), subordinatamente ad una notifica allo Stato coinvolto di almeno due mesi e sulla scorta di un programma definito con quest'ultimo (61).

Se lo scopo del questionario è sostanzialmente quello di rilevare la situazione di fatto esistente in uno Stato, attraverso le informazioni - sotto forma di risposta al questionario - che tale Stato ha fornito, non è difficile immaginare la one-site visit come uno strumento che, in una certa misura, è in grado anche di consentire un riscontro sulle informazioni pervenute, per quanto il sistema appaia comunque pensato de iure condito in un'ottica marcatamente dialogica. Inoltre, la visita è l'occasione soprattutto per instaurare tavoli di incontro con le realtà istituzionali, gli stakeholders, gli organi di informazione coinvolti nella lotta alla corruzione, cercando di raccogliere, pertanto, dalla visita una visione globale e complessiva ai fini della redazione del progetto di rapporto di valutazione. Infatti, ex art. 14 dello Statuto, il team di esperti ha il compito di redigere tale progetto preliminare di rapporto di valutazione "on the state of the law and the practice in relation to the provisions selected for the evaluation round" (62), con la collaborazione del Secretariat, il quale normalmente ne cura la parte descrittiva (basata sulle risposte al questionario e su altre informazioni raccolte), mentre è affidata al pool di esperti la redazione della parte analitica, che include invece le osservazioni e le raccomandazioni motivate.

Le Regole di Procedura prevedono che gli esperti sottopongano innanzitutto le proprie osservazioni individuali al Segretariato - entro un termine che differisce a seconda della inclusione o meno di una country visit nel Round di Valutazione (63) - al fine di provvedere alla stesura del primo Preliminary Draft Mutual Evaluation Report (c.d. P1), il quale sarà reso nella disponibilità degli esperti per eventuali osservazioni da far pervenire entro 14 giorni. All'esito di tali commenti, il Segretariato appronta il Preliminary Draft (c.d. P2), destinato non solo agli esperti ma anche allo Stato membro interessato dalla valutazione, al fine di ricevere per iscritto le sue osservazioni. I commenti espressi saranno oggetto di vaglio da parte degli esperti "with a view to determining which of them are acceptable" (64), al fine di giungere alla versione rivista del Draft Report (c.d. P3), la quale è inviata a tutti i rappresentanti che siedono nel GRECO almeno due settimane prima della Sessione Plenaria di discussione ed approvazione. La discussione in sede di Plénière è espressione evidente di quella mutualità più volte già sottolineata che permea non solo il procedimento di valutazione ma anche il controllo degli impegni assunti, attraverso il confronto diretto con tutti i membri del GRECO in ordine alle rispettive situazioni nazionali (65). Durante le plenarie, difatti, i Paesi hanno la possibilità di monitorare le situazioni e i progressi degli altri Stati, determinando le modifiche che dovessero emergere dalla discussione, ma riuscendo in qualche misura anche a valutare in un'ottica comparatistica la propria realtà domestica. All'esito della discussione, viene adottato il Report ed è data facoltà ai rispettivi Paesi interessati dalle valutazioni all'ordine del giorno della Plenaria di esprimere il loro assenso o meno alla pubblicità del rapporto (66).

Come si anticipava, il procedimento di valutazione si caratterizza per una seconda fase, in cui il GRECO, in buona sostanza, valuta se le raccomandazioni espresse nel Rapporto di Valutazione - a prescindere dalla sua pubblicazione - siano state efficacemente attuate. Tale procedura di conformità si finalizza in un rapporto sulla situazione nazionale (Compliance Report) che trae origine dal Situation Report che ogni Stato valutato è tenuto a trasmettere, in conformità all'art. 15, par. 6 dello Statuto, entro una data stabilita e 
soggetto all'approvazione dell'Assemblea plenaria, al fine di dar conto delle misure adottate per ottemperare alle raccomandazioni contenute nell'Evaluation Report (67). La prassi ci conduce a poter affermare che le raccomandazioni (68) - il più delle volte consistenti nell'individuazione di una strategia piuttosto che nell'orientamento verso il rafforzamento di taluni istituti od organi ma anche nell'indicazione di un percorso legislativo di riforme - risultano tendenzialmente prese in considerazioni dagli Stati, sebbene a ciò non (sempre) corrisponda un fedele e pedissequo allineamento alle indicazioni ricevute. D'altro canto, il sistema di risposta all'inadempimento delle raccomandazioni (69), pur previsto, può condurre in extremis all'adozione di un Public Statement che denunci la condotta dello Stato rispetto agli impegni assunti, il quale sia rimasto passivo o abbia adottato misure non sufficienti ("remains passive or takes insufficient action" (70)), il cui indiscusso valore politico fa da contraltare alla sua efficacia concreta.

\section{COVID-19 e rischi corruttivi}

Larga parte dell'operato del Gruppo si sostanzia nel supportare gli Stati ad identificare le lacune nelle national anti-corruption policies, suggerendo gli indirizzi correttivi e gli orientamenti risolutivi più appropriati. Ancor più in costanza di una fase emergenziale straordinaria - quale quella legata alla diffusione pandemica del virus SARS-CoV-2 (71) - il GRECO ha avvertito l'esigenza di richiamare gli Stati sulla necessità di non sottovalutare i rischi corruttivi ad essa connessi (72).

L'attuale contingenza economica e sociale a livello globale è eziologicamente influenzata dall'ancora persistente emergenza sanitaria internazionale. La risposta al virus ha aperto inevitabilmente nuovi spazi ed opportunità per la corruzione (73), dovuti ad un certo indebolimento dei controlli e ad una trasparenza inadeguata, distogliendo finanziamenti nel momento in cui le persone ne hanno maggiormente bisogno, all'interno di un quadro emergenziale in cui gli Stati si trovano ad agire con estrema rapidità (rectius frettolosità). A ciò, si aggiunge - come evidenziato anche dallo stesso Segretario Generale delle Nazioni Unite (74) - anche la collusione diffusa tra coloro che controllano le catene di fornitura, causando un innalzamento intollerabile dei costi di beni necessari, che distorce il mercato e inibisce spesso l'accesso a terapie salvavita. Inoltre, la pandemia in rapido sviluppo e la conseguente recessione economica hanno portato ad una forte concorrenza per le risorse essenziali. La ingente e rapida mobilitazione di risorse e fondi pubblici (vuoi per l'assistenza sanitaria vuoi per la conseguente ripresa economica) ha generato notevoli opportunità di proliferazione dei fenomeni corruttivi. Di conseguenza, la mancanza di trasparenza, la scarsa supervisione ed implementazione di misure anti-corruzione idonee e funzionali (che inevitabilmente comportano un dilatamento dei tempi vs. la tempestività necessaria dettata dall'emergenza sanitaria) hanno esacerbato tale proliferazione, creando il terreno idoneo per una wave of corruption-related incidents, e le misure pubbliche contro questi reati non hanno tenuto il passo con l'evoluzione della crisi (75).

Invero, bisogna anche considerare la inevitabile urgenza nello sviluppare ed assicurare un'affidabile, sicura ed efficace produzione vaccinale su scala mondiale, che ha condotto alla rapida approvazione di una serie di vaccini, saltando o riducendo il canonico iter di approvazione. La rapida corsa degli Stati, anche nella fase della ricerca e dello sviluppo, prima ancora che in quella dell'approvvigionamento, ha creato anche qui ulteriori opportunità per la criminalità corruttiva latu sensu intesa. Basti pensare, ad esempio, a 
tutti i potenziali conflitti di interesse legati ai finanziamenti della ricerca, nel processo di acquisto e di distribuzione di un vaccino per il COVID-19 (76).

Ebbene, in tale contesto, il GRECO ha enfatizzato la centralità degli anti-corruption and governance tools - a partire dalle due Convenzioni (civile e penale) sulla corruzione e dai Principi Guida sviluppati nel quadro del $\mathrm{CoE}$ - quantomai decisivi nell'affrontare i diversificati spazi per la criminalità corrutiva nell'ampio quadro dei pandemic-related processes. Infatti, con le guidelines a firma del Presidente (77), il Gruppo ha inteso affrontare tali questioni, sia in termini di analisi dei rischi strettamente connessi alla contingenza emergenziale, che in termini di raccomandazioni specifiche per gli Stati, sulla scorta di una serie di aree specifiche di intervento ove maggiori sono individuati $i$ relativi rischi.

In un tempo di straordinarie circostanze legate al COVID-19, in cui è il settore sanitario - maggiormente esposto (si pensi soltanto al bisogno rapido di forniture mediche vs. semplificazione degli appalti pubblici) - ad essere individuato come il destinatario bisognevole delle "cure", il GRECO pone l'accento su tre pilastri fondamentali su cui ruota l'intero assetto politico/giuridico dell'anticorruzione: accountability, oversight e transparency (78).

In particolare, il GRECO evidenzia le aree (corruzione nel sistema di approvvigionamento, servizi medici e di ricerca e sviluppo di nuovi prodotti, conflitti di interesse e lobbying, frodi), dove i rischi sono maggiormente presenti e dove, pertanto, si ritiene necessario intervenire adeguatamente.

Il rafforzamento della trasparenza nel quadro dei sistemi di approvvigionamento, quale uno degli aspetti essenziali, in termini soprattutto di prevenzione, nella lotta alla corruzione, è più volte richiamato dal GRECO, indirizzando gli Stati alla necessità di applicare con fermezza il principio-guida n. 14 dei Guiding Principles in tema di trasparenza delle procedure pubbliche di appalto (79), senza trascurare il profilo delle garanzie di un rimedio effettivo e del risarcimento per le persone fisiche e giuridiche che hanno subito un danno da un atto di corruzione (80).

Tuttavia, i rischi corruttivi possono annidarsi soprattutto nei medical-related services, laddove, con le strutture sanitarie in affanno, tra esigenze di forniture e di personale e bisogni legati all'emergenza, spesso confliggenti, la corruzione "may become part of the equation" (81). In tale precipuo ambito, il GRECO richiama gli Stati alla criminalizzazione della corruzione attiva e passiva nel settore privato, che, nel quadro della Convenzione penale sulla corruzione, compre anche gli operatori della sanità privata (82). L'analisi del GRECO prende anche in considerazione la micro-corruzione (petty bribery) - "an issue that has emerged again in the pandemic context" (83) - in tale settore particolarmente presente, ad esempio, per l'accesso ai servizi medici, ai test e alle attrezzature, alle procedure di raccolta e sepoltura del corpo, per la quarantena, anche in quei Paesi dove tale tipologia di corruzione era sinora davvero residuale.

Come già anticipato, terreno fertile risultano essere anche i notevoli investimenti in research and development, dovuti alla corsa alla cura e al vaccino, rispetto cui il GRECO enfatizza la necessità di incrementare la "capacity, authority and public accountability of State institutions" (84), incaricate di quelle funzioni di controllo e regolamentazione la cui implementazione è ancor più determinante in un contesto emergenziale di tale portata. Il rafforzamento di tali funzioni può avere un ruolo decisivo anche rispetto ai conflitti di interesse inevitabilmente generati in un tale contesto. Infatti, il GRECO affronta tale tema attraverso il richiamo alle sue raccomandazioni sul reclutamento e la promozione merit- 
based di funzionari apicali e personale nonché sui codici di condotta (85) e i meccanismi di prevenzione/integrità del conflitto di interesse (86). Anche qui, inoltre, il richiamo del GRECO si proietta verso l'essenzialità della trasparency, al fine di garantire il corretto funzionamento dei processi decisionali, ponendosi al riparo dalle attività lobbistiche, anche sotto il profilo della disseminazione delle informazioni (87).

Non da ultimo, il GRECO evidenzia i rischi collegati alle frodi e al conseguente riciclaggio dei proventi, con la proliferazione delle falsificazioni di prodotti sanitari, indispensabili non solo per le strutture ospedaliere e di cura, ma anche per i privati cittadini, dato l'incremento straordinario della domanda di dispositivi di protezione individuale come di altre forniture mediche, a cui è corrisposto un aumento dell'offerta di tali prodotti su falsi siti web, shop online, social accounts, emails (phishing) (88). Riguardo tali aspetti, l'appello del GRECO è di rinvio alla Convenzione del Consiglio d'Europa sulla contraffazione dei prodotti medicali e reati simili che implicano una minaccia alla salute pubblica (c.d. Convenzione MEDICRIME) del 2011 (89), quindi alla criminalizzazione di quelle fattispecie di produzione, fornitura, offerta e traffico di prodotti contraffatti, nonché di falsificazione di documenti e di altri reati affini (90).

È interessante notare che il citato documento, emanato dal Presidente del GRECO, non dimentica - sebbene attraverso un cenno marginale - la rilevanza dell'impatto sui diritti umani, la democrazia e lo Stato di diritto (91), al cui rispetto gli Stati sono sempre chiamati, a maggior ragione nel contesto di una legislazione emergenziale e delle conseguenti forme di devolution of powers. In particolare, il GRECO porta l'attenzione degli Stati verso la protezione delle persone che segnalano le condotte corruttive (92) e il cui valore è, pertanto, essenziale "in deterring and preventing wrongdoing, and in strengthening democratic accountability and transparency" (93).

L'attenzione del documento è, infine, dedicata al settore privato, dove pure i rischi corruttivi possono trovare ampi spazi fertili (e.g., tangenti per facilitare la progressione di processi bloccati a causa della carenza di personale o chiusura di uffici pubblici, falsificazione di documenti per soddisfare le condizioni dei regimi di aiuti di Stato per le misure di sollievo dalla pandemia, scavalcamento dei requisiti di certificazione dei prodotti, donazioni, mancanza di risorse per supervisionare la cattiva condotta da parte dei dipendenti), e sui cui il quadro di riferimento cui ispirarsi, nelle indicazioni del GRECO, è rappresentato dagli artt. 7 e 8 della Convenzione penale sulla corruzione, nonché il principio-guida n. 5 dei Guiding Principles (94), oltre alle raccomandazioni per il settore privato contenute nel Secondo Evaluation Round.

\section{Osservazioni conclusive}

Il contributo del $\mathrm{CoE}$ alla lotta alla corruzione ha dimostrato, nel tempo, di possedere quei caratteri di multidisciplinarietà, poliedricità e complementarietà tali da fornire all'intero spazio giuridico europeo una serie di strumenti - siano essi di carattere perentorio o incardinati piuttosto in forme di moral suasion - idonei a costruire un sistema organico di norme internazionali a disposizione degli Stati per incidere positivamente su quel "fenomeno criminale e immorale" (95) della corruzione, che "mette alla prova le nostre società e le nostre economie" (96) e, inoltre, "ostacola la crescita economica sostenibile, distorce la concorrenza sul mercato, mina lo stato di diritto e la fiducia tra cittadini e governi" (97). 
Inoltre, la creazione di un sistema di reciproca valutazione, quale quello che ruota intorno al GRECO - che è innanzitutto uno strumento di confronto e di dialogo interstatale ed interordinamentale, prima ancora che un meccanismo "giudicante" - si è dimostrata una felice intuizione. Nel tempo, esso ha dato prova della sua attitudine a tradurre obiettivi in realizzabilità concreta, anche tra contesti normativi sovente assai diversi fra loro.

Certamente, è necessario tener presente che il sistema dipende anche (e soprattutto) dalla effettiva ricettività degli Stati agli impulsi raccomandatori derivanti da Strasburgo, non essendo il GRECO dotato di un sostanziale meccanismo di reazione agli inadempimenti statali, tale da poter intervenire sulla reticenza in maniera performante. Tuttavia, l'esperienza dopo cinque Round valutativi ha, nel complesso, offerto un quadro di compliance soddisfacente, potendo registrare un trend positivo per molti Stati verso lo sviluppo, l'implementazione e l'innovazione delle misure anticorruzione nei rispettivi ordinamenti (98).

La lotta alla corruzione, quale minaccia globale, riveste senz'altro un ruolo cruciale, che si avvale del positivo apporto del Consiglio d'Europa, ma che necessità quanto mai di un rinnovato multilateralismo efficiente e di un'efficace cooperazione internazionale al fine di rafforzare i sistemi giuridici nazionali, messi a durissima prova dalla pandemia di COVID-19, che ha fatto precipitare la comunità internazionale nella peggiore crisi globale dal dopoguerra. Come ha, difatti, evidenziato anche il GRECO nel suo ultimo rapporto annuale (99), ormai da oltre un anno i governi hanno dovuto istituire misure di emergenza che hanno implicato la concentrazione di poteri e deroghe ai diritti fondamentali, misure che vanno di pari passo con rischi di corruzione da non sottovalutare. Perciò, è fondamentale che, in situazioni di emergenza, tutte le decisioni e le procedure si ispirino ai principi di trasparenza, integrità e responsabilità, divenendo cruciale un'intensificazione degli sforzi per assicurare che tutte le politiche e le azioni volte ad affrontare le crisi in ambito economico e sanitario rispettino le norme anticorruzione.

Dall'analisi dei rischi corruttivi, emersi dalla necessità di adottare misure straordinarie per combattere la pandemia da COVID-19, tra cui fra tutte l'iniezione di grandi quantità di denaro nell'economia per affievolirne l'impatto economico e sociale, traspare un quadro senz'altro preoccupante, rispetto cui non solo si rende necessaria l'adozione di adeguati interventi legislativi, soprattutto sul fronte preventivo, per contrastare la corruzione ma diventa essenziale che tali norme siano effettivamente applicate nella pratica e i governi agiscano con trasparenza e senso di responsabilità. Prevenire la corruzione di fronte alla crisi e tenerla sotto controllo, è tanto arduo quanto essenziale, onde evitare che essa paralizzi l'efficacia delle risposte all'emergenza (soprattutto in quei Paesi dove non sono già stati predisposti solidi meccanismi di anticorruzione) (100).

Dunque, con un occhio attento alle nuove aree di rischio, come lo è quella delle crisi, occorre misurarsi con la corruzione come un fenomeno sofisticato e variegato, che vive in una sua dimensione sempre più fluida, diffusa, interconnessa e che necessita di una risposta globale.

La decisione di tenere una Sessione speciale dell'Assemblea Generale delle Nazioni Unite contro la corruzione (101), dalla quale sono scaturiti una serie importanti ed ambiziosi impegni "to effectively addressing challenges and implementing measures to prevent and combat corruption and strengthen international cooperation", sintentizzati nella Dichiarazione politica in seno ad essa adottata (102), unitamente alla spinta dell'agenda globale anticorruzione del G20 (103) (a presidenza italiana) verso una nuova 
era di enforcement, cui sta lavorando il Gruppo di lavoro anticorruzione (ACWG) del G20 (104), sono forse il segno di un rinnovato approccio verso un'azione rafforzata e coordinata per prevenire e contrastare la corruzione soprattutto attraverso l'elaborazione di politiche e strategie comuni e condivise nei fora multilaterali.

In tale prospettiva, ben si colloca la mission e il ruolo del GRECO, come sede elettiva di confronto e di discussione, ma anche di promozione ed attuazione concreta di misure idonee ed efficaci, che possano contribuire in maniera determinante all'indebolimento del fenomeno corruttivo.

\section{References}

(1) Per un inquadramento generale, sulla corruzione v. G. TARTAGLIA POLCINI, Il contrasto alla corruzione nell'attività dei fori multilaterali: La Presidenza italiana del G20, in T. RUSSO, A. ORIOLO, (a cura di), La lotta contro la corruzione nella legalità reticolare: il sistema penale multilivello, Salerno, (in corso di pubblicazione) 2021; L. BORLINI, "Not such a retrospective": riflessioni sull'origine, sviluppo e conseguimenti della cooperazione internazionale anti-corruzione, in DPCE online, Vol. 38, $\mathrm{n}$. 1, 2019; N. PARISI, La prevenzione della corruzione nel modello internazionale ed europeo, in Federalismi.it, n. 9, 2019; N. PARISI, G.L. POTESTÀ, D.G. RINOLDI (a cura di), Prevenire la corruzione. Questioni e modelli emergenti tra diritto, etica ed economia, Napoli, 2018; N. PARISI, Il contrasto alla corruzione e la lezione derivata dal diritto internazionale: non solo repressione, ma soprattutto prevenzione, in Diritto comunitario e degli scambi internazionali, n. 2-3, 2016, pp. 185-210; M. ARNONE, L. BORLINI (eds.), Corruption. Economic Analysis and International Law, Cheltenham-Northampton, 2014; A. SARAVALLE, Corruzione internazionale e "ne bis in idem", in F. BONELLI, M. MANTOVANI (a cura di), Corruzione nazionale e internazionale, Milano, 2014; E. ZANIBONI, Ordine internazionale e lotta alla corruzione, in Diritto pubblico comparato ed europeo, n. 4, 2014; L. BORLINI, Corruzione. Impatto sistemico e normativa internazionale, in C. D'ARGENTINE BERIA (coord.), Riciclaggio e corruzione: prevenzione e controllo tra fonti interne e internazionali, Milano, 2013; V. MONGILLO, La corruzione fra sfera interna e dimensione internazionale, Napoli, 2012; L. BORLINI, P. MAGRINI, La lotta alla corruzione internazionale. Dall'ambito OCSE alla dimensione ONU, in Diritto del commercio internazionale, Vol. 21, Issue 1, 2007, pp. 15-127; N. PARISI, D.G. RINOLDI, Pluralità di strumenti pattizi internazionali di lotta alla corruzione e adattamento dell'ordinamento italiano, in G. SACERDOTI (a cura di), Responsabilità d'impresa e strumenti internazionali anticorruzione, Milano, 2003, pp. 254-273. Sotto il profilo più marcatamente italiano, si veda N. PARISI, L'attività di contrasto alla corruzione sul piano della prevenzione. A proposito di appalti, ma non solo..., in R. BORSARI (a cura di), La corruzione a due anni dalla "Riforma Severino", Padova, 2016, pp.91-136; M. NINO, L'applicazione delle convenzioni internazionali in materia di contrasto alla corruzione internazionale nell'ordinamento italiano, in La Comunità internazionale, Vol. LXVIII, n. 3, 2013, pp. 489-515; N. PARISI, D.G. RINOLDI, L'applicazione in Italia di strumenti giuridici internazionali contro la corruzione, G. FORTI (a cura di), Il prezzo della tangente. La corruzione come sistema a dieci anni da "mani pulite", Milano, 2003, pp. 191231.

Sul piano interno, più squisitamente penalistico, v. L. FOFFANI, La nuova dimensione internazionale ed economica della lotta alla corruzione: dal settore pubblico al settore privato, in A. ORIOLO, T. RUSSO (a cura di), op. cit., p.; G. DALIA, L'esperienza italiana nella lotta alla corruzione: prevenzione, sanzione penale, contrasto processuale e performance, in Iura and Legal Systems, Vol. 6, 2019, pp.1-31; R. CANTONE, Il contrasto alla corruzione. Il modello italiano, in Diritto Penale Contemporaneo, 2018; A. SESSA, Sistema penale e "legalità reticolare": opzioni di valore nella più recente normativa anticorruzione, in Le Corti Salernitane, Vol. 1-2, 2017, pp. 261-293; G.B. MATTARELLA, M. PELISSERO (a cura di), La legge anticorruzione. Prevenzione e repressione della corruzione, Torino, 2013.

(2) Sul piano comunitario, si veda L. BORLINI, Regulating Criminal Finance in the EU in the Light of the International Instruments, in Yearbook of European Law, Vol. 36, 2017, pp. 553-598; L. BORLINI, F. MONTANARO, The Evolution of the EU Law Against Criminal Finance: The "Hardening" of FATF Standards Within the EU, in Georgetown Journal of International Law, Vol. 48, 2017, pp. 1009-1062; V. 
MONGILlO, op. cit., p. 463 ss.; C.R. CALDERONE, La lotta alla corruzione in campo comunitario ed internazionale, in Rivista trimestrale di diritto penale dell'economia, Vol. 14, Fasc. 3, 2001, 607-619; S. MANACORDA, Corruzione internazionale e tutela penale degli interessi comunitari, in Diritto penale e processo, Vol. 7, Fasc. 4, 2001, pp. 410-428.

(3) F. LONGO (a cura di), L'Unione Europea e il "cerchio di amici". Sicurezza europea e politica di vicinato, Milano, 2008, p.XIII.

(4) Di strumenti giuridici "regionali", ne abbiamo contezza diffusamente anche in ambito extra-europeo. È bene, tra questi, menzionare, in una elencazione certamente non esaustiva: la Convenzione interamericana contro la corruzione, adottata il 29 marzo 1996 dall'Organizzazione degli Stati americani (OAS), ed entrata in vigore il 6 marzo 1997; il Protocollo contro la corruzione adottato dalla Comunità di Sviluppo dell'Africa Meridionale (SADC) il 14 agosto 2001; il Protocollo sulla lotta alla corruzione della Comunità economica degli Stati dell'Africa occidentale (ECOWAS Protocol), firmato il 21 dicembre 2001; la Convenzione sulla prevenzione e la lotta alla corruzione emanata dall'Unione Africana 1'11 giugno 2003, ed entrata in vigore il 5 agosto 2006 .

(5) Istituito con il Trattato di Londra (Statuto del Consiglio d'Europa, STE n. 001), aperto alla firma il 5 maggio 1949 ed entrato in vigore il 3 agosto 1949, i cui Paesi fondatori furono Belgio, Danimarca, Francia, Irlanda, Italia, Lussemburgo, Paesi Bassi, Norvegia, Svezia e Regno Unito.

(6) Art. 1(a), Statuto del Consiglio d'Europa.

(7) Cfr. le interessanti considerazioni di N. PARISI, Il ruolo dell'Autorità nazionale anticorruzione. Una prospettiva sistematica in disaccordo con la vulgata opinio, in DPCE online, 2020/4, pp. 4631-4667, specific. p. 4632, la quale evidenzia come "gli Stati hanno anzitutto preso atto dell'inadeguatezza e dell'insufficienza di una strategia organizzata sul solo terreno delle norme di diritto penale sostanziale e procedurale", aggiungendo, inoltre, che "la repressione si era nei fatti dimostrata insufficiente a evitare che si radicasse il fenomeno, diffuso e pervasivo". Perciò, secondo l'A., "di fronte a un fenomeno il diritto penale non è lo strumento adatto al suo contrasto: esso - rappresentando l'extrema ratio in uno Stato di diritto e in questo manifestando la propria sussidiarietà - deve essere riservato a casi eccezionali".

(8) V. G. LOCATI, Le convenzioni del Consiglio d'Europa in materia di lotta alla corruzione e gli adempimenti nazionali di alcuni stati ad esse aderenti, in Liuc Papers, n. 123, 2003.

(9) Gruppo Multidisciplinare sulla Corruzione, Programme of Action Against Corruption, doc. GMC (95) 49, 25-27 settembre 1995. Il Programma è stato, poi, successivamente, adottato dal Comitato dei Ministri al suo 578esimo meeting (doc. CM (96) 133 and Addendum), tenutosi dal 18 a 21 novembre 1996.

(10) Convenzione penale sulla corruzione, STE n. 173, aperta alla firma il 27 gennaio 1999 ed entrata in vigore il $1^{\circ}$ luglio 2002 con 14 ratifiche. Vale la pena precisare che il Trattato era aperto alla firma tanto degli Stati membri quanto degli Stati non membri i quali avevano comunque partecipato alla sua elaborazione ed era, inoltre, aperto all'adesione degli altri Stati non membri e dell'Unione europea.

(11) Protocollo addizionale alla Convenzione penale sulla corruzione, STE n. 191, aperto alla firma il 15 maggio 2003 ed entrato in vigore il $1^{\circ}$ febbraio 2005. Il Protocollo vanta attualmente soltanto 5 ratifiche, rispetto al numero ben maggiore di Stati che hanno firmato il relativo Trattato.

(12) Convenzione civile sulla corruzione, STE n. 174, aperta alla firma il 4 novembre 1999 ed entrata in vigore il $1^{\circ}$ novembre 2003 con 14 ratifiche. Anche in questo caso, la firma e l'adesione erano aperte in maniera analoga alla "sorella" penale.

(13) V. W. RAU, Group of States Against Corruption (GRECO), in S. SCHMAHL, M. BREUER (eds.), The Council of Europe. Its Law and Policies, Oxford, 2017, pp. 444-460; B. RAZAVI FARD, H. HASSANPOUR, Reviewing the Strategies of Dealing with Corruption in the Europe Union Countries with Regulatory System of the Group of States Against Corruption (GRECO), in Journal of Politics and Law, Vol. 9, No. 5, 2016, pp. 47-58; W. RAU, The Group of States Against Corruption (GRECO) - Operation and Results from its Current Third Evaluation Round, in L. ACHATHALER, D. HOFMANN, M. PÁZMÁNDY (eds.), Korruptionsbekämpfung als globale Herausforderung, Wiesbaden, 2011, pp. 19-36. (14) Non vogliano dimenticarsi, tra le fonti, anche i Venti Principi Guida contro la Corruzione (Risoluzione 97/24 del Comitato dei Ministri del Consiglio d'Europa, Twenty Guiding Principles Against Corruption, del 6 novembre 1997); La Raccomandazione sul Codice di Condotta per i funzionari pubblici (Raccomandazione del Comitato dei Ministri del Consiglio d'Europa, on Codes of Conduct for Public Officials, dell'11 maggio 2000, R(2000)10); la Raccomandazione sulle regole comuni sulla Corruzione nel finanziamento ai partiti e nelle campagne elettorali (Raccomandazione del Comitato dei Ministri del Consiglio d'Europa, on Common Rules Against Corruption in the Funding of Political Parties and Electoral Campaigns, dell' 8 aprile 2003, R(2003)4). 
(15) Gruppo di Stati contro la Corruzione, Corruption Risks and Useful Legal References in the Context of COVID-19, del 15 aprile 2020, Greco(2020)4.

(16) H. FURRER, La dimensione paneuropea del Consiglio d'Europa, in Rivista di Studi Politici Internazionali, Vol. 64, No. 4, 1997, pp. 510-534.

(17) N. PARISI, Il ruolo dell'Autorità nazionale anticorruzione. Una prospettiva sistematica in disaccordo con la vulgata opinio, cit., p. 4633.

(18) H. FURRER, op. cit., p. 511.

(19) Preambolo, Statuto del Consiglio d'Europa.

(20) Invero, già a partire dal 1981, con la Raccomandazione del Comitato dei Ministri del Consiglio d'Europa, on Economic Crime, del 25 giugno 1981, R(81)12, il Comitato aveva iniziato a compulsare gli Stati nell'intraprendere azioni ed iniziative nei confronti della criminalità economica, inclusa la corruzione. (21) 19esima Conferenza dei Ministri della Giustizia europei, La Valletta (Malta), 14-15 giugno 1994. V. Risoluzione n. 1 della Conferenza dei Ministri della Giustizia europei, on Civil, Administrative and Criminal Law Aspects of Corruption, del 14-15 giugno 1994.

(22) Ivi, par. 1.

(23) Programme of Action Against Corruption, cit., p.14.

(24) Ivi, p.15.

(25) Risoluzione 97/24 del Comitato dei Ministri del Consiglio d'Europa, cit., nota 14.

(26) Ivi, Principio n. 2.

(27) Ivi, Principio n. 7.

(28) Ivi, Principio n. 9.

(29) Ivi, Principio n. 20.

(30) V. G. LOCATI, op.cit.

(31) Raccomandazione R(2000)10, cit., nota 14.

(32) Raccomandazione R(2003)4, cit., nota 14.

(33) In tal senso, l'art. 3 della Raccomandazione R (2000) 10 definisce chiaramente che "the purpose of this Code is to specify the standards of integrity and conduct to be observed by public officials, to help them meet those standards and to inform the public of the conduct it is entitled to expect of public officials".

(34) Convenzione sulla lotta alla corruzione di pubblici ufficiali stranieri nelle operazioni economiche internazionali, firmata a Parigi il 17 dicembre 1997, entrata in vigore il 15 febbraio 1999, firmata e ratificata dai 38 Stati membri dell'OCSE, oltre che da 6 Stati non membri (Argentina, Brasile, Bulgaria, Perù, Russia e Sudafrica).

(35) È bene evidenziare che tale distinzione attiene alle dinamiche dell'illecito corruttivo in riferimento ai soggetti agenti, per cui la prima si configura con il fatto del privato (extraneus) che dà o promette un'utilità al soggetto pubblico quale corrispettivo per un atto d'ufficio, mentre la seconda si ha qualora il soggetto pubblico (intraneus) accetti dal privato un'utilità non dovuta, o comunque ne accetta la promessa, al fine di compiere, omettere o ritardare l'atto.

(36) Preambolo, Convenzione civile sulla corruzione.

(37) Per una panoramica sul whistleblowing, v. T. RUSSO, Il whistleblowing come strumento "multilivello" nella lotta alla corruzione, in T. RUSSO, A. ORIOLO (a cura di), op. cit.; D.G. RINOLDI, N. PARISI, G. FRASCHINI (a cura di), Il whistleblowing. Nuovo strumento di lotta alla corruzione, Catania, 2011.

(38) Art. 1, Convenzione civile sulla corruzione.

(39) Tale esperienza è stata già preceduta, nel quadro OCSE, dall' OECD Working Group on Bribery in International Business Transactions, fondato nel 1994 e responsabile del monitoraggio dell'implementazione e dell' enforcement della Convenzione OCSE sulla lotta alla corruzione di pubblici ufficiali stranieri nelle operazioni economiche internazionali del 1997, oltre che della Raccomandazione del Consiglio OCSE, for Further Combating Bribery of Foreign Public Officals in International Business Transactions, del 26 novembre 2009. Inoltre, si rammenta anche il più recente peer review process che, in ambito onusiano, è stato creato per monitorare l'applicazione della Convenzione delle Nazioni Unite contro la corruzione (UNCAC) del 2003, ovvero l'UNCAC Review Mechanism, istituito con la Risoluzione 3/1 della Conferenza degli Stati parte della Convenzione delle Nazioni Unite contro la corruzione, 3-6 novembre 2009. A tale ultimo proposito, si veda S. BUSILLO, Asset recovery: nuova enfasi da parte delle nazioni unite nella lotta alla corruzione, in T. RUSSO, A. ORIOLO, G. DALIA (eds.), "EU-Western Balkans Cooperation on Justice and Home Affairs", 2nd Edition Essays, OPTIME, (in corso di pubblicazione) 2021.

(40) Art. 1, Statuto del GRECO. 
(41) Secondo Summit del Consiglio d'Europa, Strasburgo, 10-11 ottobre 1997.

(42) Secondo Summit del Consiglio d'Europa, Action Plan, dell'11 ottobre 1997, sez. III, par. 2.

(43) Risoluzione 98/7 del Comitato dei Ministri del Consiglio d'Europa, Authorising the Partial and Enlarged Agreement Establishing the "Group of States against Corruption - GRECO", del 5 maggio 1998. (44) Risoluzione 99/5 del Comitato dei Ministri del Consiglio d'Europa, Establishing the "Group of States Against Corruption - GRECO”, del $1^{\circ}$ Maggio 1999.

(45) Risoluzione 99/5 del Comitato dei Ministri del Consiglio d'Europa, Appendix to Resolution (99) 5 , Statute of the Group of States Against Corruption (GRECO), del $1^{\circ}$ Maggio 1999.

(46) Gruppo di Stati contro la Corruzione, Rules of Procedure, Prima Sessione plenaria, Strasburgo, 4-6 ottobre 1999. Tali Regole sono poi state successivamente emendate ben dieci volte. Da ultimi, vi sono gli emendamenti adottati durante la 76esima Sessione plenaria del 19-23 giugno 2017 a Strasburgo.

(47) Art. 4, parr. 1-2-3, Statuto del GRECO.

(48) Una norma ad hoc, invece, regola l'adesione della (allora) Comunità europea, la quale è ammessa all'adesione soltanto attraverso una Risoluzione-invito del Comitato dei Ministri, che ne deve esprimere anche le modalità di partecipazione (Art. 5, Statuto del GRECO; Regola n. 2, Regole di Procedura del GRECO). Attualmente, vale la pena precisare che l'Unione europea, dal 10 luglio 2019, gode del mero stato di Osservatore.

(49) Art. 4, par. 4, Statuto del GRECO.

(50) Artt. 32-33 di entrambe le Convenzioni citate.

(51) In particolare, l'art. 6 dello Statuto del GRECO si esprime nel senso che "each member shall appoint a delegation to the GRECO consisting of not more than two representatives", aggiungendo poi che "one representative shall be appointed as head of the delegation".

(52) Art. 10, par. 4, Statuto del GRECO.

(53) Il Bureau, ai sensi dell'art.9 dello Statuto, ha il compito di: i) preparare la bozza preliminare del programma annuale di attività e la bozza del report annuale delle attività; ii) formulare proposte al GRECO riguardo la bozza di budget preliminare; iii) organizzare le visite nei Paesi sulla base delle decisioni prese dal GRECO; iv) formulare proposte al GRECO sulla composizione dei teams di valutazione ad hoc; v) preparare l'agenda delle Sessioni del GRECO, incluse quelle in cui saranno discussi i report di valutazione; vi) formulare proposte al GRECO con riguardo alle regole da stabilire per la procedura di valutazione ex art.10, par.3, dello Statuto; vii) formulare proposte al GRECO con riguardo alla nomina degli esperti scientifici e dei consulenti; viii) svolgere ogni altra funzione ad esso specificamente assegnata dal GRECO. (54) Art. 9, par. 1, Statuto del GRECO.

(55) La Statutory Committee è sostanzialmente l'organo "economico-finanziario" del GRECO e ha, invece, il compito di determinare ogni anno l'ammontare del contributo economico obbligatorio dovuto, oltre che di approvare il budget, secondo le modalità definite dall'art.18 dello Statuto del GRECO. Tuttavia, l'organo ha un rilevante peso perché lo Statuto gli affida talune decisioni rilevanti nel quadro del funzionamento del GRECO (art. 8, parr. 1 e 6; art. 16; art. 17, par. 4) oltre al fatto che un suo rappresentante siede anche nel GRECO.

(56) Art. 19, Statuto del GRECO; Regola n. 10, Regole di Procedura del GRECO.

(57) L'art. 10, par. 2, dello Statuto del GRECO ne esprime nitidamente la definizione, descrivendo un ciclo di valutazione come "a period of time determined by the GRECO, during which an evaluation procedure shall be conducted to assess the compliance of members with selected provisions contained in the Guiding Principles and in other international legal instruments adopted in pursuance of the Programme of Action against Corruption".

(58) Art. 1, Statuto del GRECO; Regola n. 22, par. 2, Regole di Procedura del GRECO.

(59) Art.11, Statuto del GRECO; Regola n. 24, Regole di Procedura del GRECO.

(60) Art. 13, Statuto del GRECO.

(61) È opportuno precisare che la Regola n. 27 delle Regole di Procedura del GRECO definisce più nel dettaglio il quadro operativo e regolamentare entro il quale deve avere luogo la visita. In esso, si prescrive, in particolare, la necessità che la visita non abbia luogo prima di 3 mesi dalla ricezione delle risposte al questionario da parte del Segretariato (par. 2) e, inoltre, che la sua lunghezza non superi i 4 giorni (con possibilità di estensione "in special cases" da parte del Bureau o del Secretariat), secondo un cronoprogramma ben definito (par. 5).

(62) Art. 14, Statuto del GRECO.

(63) Il termine de qua è di 14 giorni dalla fine della visita nel Paese, mentre, in assenza di visita, lo stesso decorre invece dal ricevimento della bozza contenente la parte descrittiva redatta dal Segretariato.

(64) Regola n. 28, par. 8, Regole di Procedura del GRECO. 
(65) G. LOCATI, op. cit., p. 7.

(66) Art. 15, par. 5, dello Statuto del GRECO: "Evaluation reports shall be confidential. Unless otherwise decided, access to these reports shall be restricted to members of the team which has carried out the evaluation, in addition to members of the GRECO, of the Statutory Committee and of the Secretariat of these bodies". Di norma, alla chiusura della seduta plenaria, gli Stati valutati non si esprimono subito a favore della pubblicazione del rapporto che li riguarda, ma l'autorizzazione alla pubblicazione interviene in un momento successivo.

(67) Regola n. 30, par. 2, Regole di Procedura del GRECO: "In conformity with article 15, paragraph 6, of the Statute members shall, within 18 months after the adoption of the evaluation report, address to GRECO a situation report (...) indicating the measures taken to follow the recommendations in the evaluation report. GRECO will examine these reports and decide whether or not the recommendations have been complied with".

(68) Il GRECO è attualmente giunto al quinto Round di Valutazione (ancora in corso), lanciato il 20 marzo 2017 e avente ad oggetto il tema della prevenzione della corruzione e della promozione dell'integrità nei Governi e nelle forze di polizia.

(69) A tal proposito, la Regola n. 31 delle Regole di Procedura prevede che in caso di non ottemperanza parziale o totale alle raccomandazioni del GRECO i membri dello stesso, riuniti in seduta plenaria, alla luce di tali rilievi, possano adottare misure volte a convincere lo stato a conformarsi a quanto previsto, innanzitutto sollecitando la produzione di un rapporto ulteriore e scadenzato (c.d. RC2) da parte dello stato de quo, a cui può seguire la richiesta di ulteriori rapporti sullo stato di avanzamento. Qualora permanga la situazione di non-compliance, Il GRECO (alternativamente o congiuntamente) informa manu propria dell'inadempienza il Capo delegazione dello Stato interessato (Regola n. 32, par. 2 (ii), lett. (a)) oppure invita il Presidente del Comitato statutario a scrivere al Rappresentante permanente dello Stato interessato presso il CoE (Regola n. 32, par. 2 (ii), lett. (b)) oppure, ancora, invita il Segretario generale del CoE ad inviare una lettera al Ministro degli Affari Esteri di quello Stato (Regola n. 32, par. 2 (ii), lett. (c)). Inoltre, in qualsiasi fase della procedura di non-compliance, il GRECO può inviare ex Regola n. 32, par. 2 (iii) delle Regole di Procedura presso lo Stato inadempiente una missione composta da funzionari di alto livello come il Presidente stesso del GRECO, il Segretario esecutivo, il Direttore generale degli affari giuridici e alcuni capi delegazione dei Paesi membri - "with a view to reinforcing the importance of complying with the relevant recommendations". In ultima istanza, se ancora lo Stato non intende adeguarsi alle richieste, il GRECO si rivolge nuovamente al Comitato statutario affinché valuti l'adozione del Public Statement di cui all'art. 16 dello Statuto.

(70) Art.16, Statuto del GRECO.

(71) La sindrome respiratoria acuta grave Coronavirus-2 (SARS-CoV-2) è il nome dato al nuovo coronavirus del 2019, che causa la malattia associata conosciuta globalmente col nome di COVID-19. Tale nuovo ceppo di coronavirus, che non è stato precedentemente identificato nell'uomo, ha iniziato a destare interesse a livello internazionale, soltanto a seguito della notifica da parte della Cina, alla fine del dicembre 2019, dell'esistenza di una polmonite da causa sconosciuta. L'Organizzazione Mondiale della Sanità ha dichiarato il nuovo coronavirus quale emergenza di sanità pubblica di rilevanza internazionale, ai sensi degli artt. 12 e 43 del Regolamento Sanitario Internazionale, il 30 gennaio 2020, riconoscendone il carattere pandemico l'11 marzo 2020.

(72) Il riferimento è, in particolare, alle guidelines del 15 aprile 2020 (v., supra, nota 15), ma anche agli anti-corruption trends, challenges and good practices emergenti dal 21esimo General Activity Report del GRECO, pubblicato nell'Aprile 2021, disponibile al link https://rm.coe.int/21st-general-activity-reportgreco-2020/1680a2173c.

(73) La dottrina, sul punto, è alquanto scarna. Si veda V. TEREMETSKYI, Y. DULIBA, V. KROITOR E AA, Corruption and Strengthening Anti-Corruption Efforts in Healthcare During the Pandemic of Covid19, in Medico-Legal Journal, Vol. 89, Issue 1, 2021, pp. 25-28.

(74) Nazioni Unite, Dichiarazione del Segretario Generale sulla corruzione nel contesto del COVID-19, 15 ottobre 2020

(75) V. S. ROSE-ACKERMAN, Corruption and Covid-19, in Eunomía. Revista en Cultura de la Legalidad, No. 20, 2021, pp. 16-36.

(76) Per un maggiore approfondimento generale, v. Ufficio delle Nazioni Unite per il controllo della droga e la prevenzione del crimine (UNODC), COVID-19 Vaccines and Corruption Risks: Preventing Corruption in the Manufacture, Allocation and Distribution of Vaccines, disponibile al link https://www.unodc.org/documents/corruption/COVID-19/Policy_paper_on_COVID-

19_vaccines_and_corruption_risks.pdf. 
(77) GRECO, Corruption Risks and Useful Legal References in the Context of COVID-19, cit.

(78) Ivi, p. 1.

(79) Venti Principi Guida per la Lotta alla Corruzione, supra, nota 14, principio-guida n. 14: "to adopt appropriately transparent procedures for public procurement that promote fair competition and deter corruptors".

(80) Il riferimento evidente qui è, invece, alla Convenzione civile sulla corruzione.

(81) GRECO, Corruption Risks and Useful Legal References in the Context of COVID-19, cit., p. 2.

(82) Artt. 7-8, Convenzione penale sulla corruzione.

(83) GRECO, Corruption Risks and Useful Legal References in the Context of COVID-19, cit., p. 3.

(84) GRECO, Corruption Risks and Useful Legal References in the Context of COVID-19, cit., p. 5.

(85) V., infra, par. 2.

(86) Si veda, da ultimo, il 21esimo General Activity Report del GRECO, cit., nota 72. Si veda, inoltre, la Raccomandazione R (2000), cit., nota 14.

(87) V., in particolare, Raccomandazione del Comitato dei Ministri del Consiglio d'Europa, Legal Regulation of Lobbying Activities in the Context of Public Decision Making, del 22 marzo 2017, R(2017)2. (88) È possibile leggere ed approfondire i rischi di finanza illecita, anche in materia di terrorismo, emergenti dalle sfide poste dal COVID-19 anche attraverso la lente d'ingrandimento di altri organismi internazionali, quali il Gruppo di Azione Finanziaria Internazionale (GAFI) e l'Organizzazione Internazionale della Polizia Criminale (INTERPOL), cui si rimanda. V. GRUPPO DI AZIONE FINANZIARIA INTERNAZIONALE, Update: COVID-19-related Money Laundering and Terrorist Financing, Parigi, Dicembre 2020; ORGANIZZAZIONE INTERNAZIONALE DELLA POLIZIA CRIMINALE, COVID-19 Pandemic Interpol Recommendations for Law Enforcement, novembre 2020; ORGANIZZAZIONE INTERNAZIONALE DELLA POLIZIA CRIMINALE, Cybercrime: COVID-19 Impact, agosto 2020; GRUPPO DI AZIONE FINANZIARIA INTERNAZIONALE, COVID-19-related Money Laundering and Terrorist Financing. Risks and Policy Responses, Parigi, maggio 2020; GRUPPO DI AZIONE FINANZIARIA INTERNAZIONALE, Statement by the FATF President: COVID-19 and Measures to Combat Illicit Financing, del $1^{\circ}$ aprile 2020.

(89) Convenzione del Consiglio d'Europa sulla contraffazione dei prodotti sanitari e reati affini che rappresentano una minaccia per la salute pubblica, n. 211, aperta alla firma il 28 ottobre 2011, entrata in vigore il $1^{\circ}$ gennaio 2016.

(90) In dottrina, si veda C.M.R. CASABONA, A.U. MORA, P.N. JIMÉNEZ, Ó. ALARCÓN-JIMÉNEZ, International Strategies in Fighting Against Medicaments Fraud and Other Similar Offences. The MEDICRIME Convention, in Crime Law and Social Change, Vol. 68, Issue 1-2, 2017, pp. 95-122; S. NEGRI, The Medicrime Convention: Combating Pharmaceutical Crimes Through European Criminal Law and Beyond, in New Journal of European Criminal Law, Vol. 7, Issue 3, 2016, pp. 350-367.

(91) Per un approfondimento sul rapporto tra corruzione e diritti umani, si veda A. ORIOLO, Corruzione e diritti umani nella giurisprudenza di Strasburgo, in T. RUSSO A. ORIOLO (a cura di), op. cit.; M. MARZANO, La corruzione come violazione dei diritti umani, in Diritto penale della globalizzazione, Fasc. 4, 2020; K. OLANIYAN, Towards a Human Rights Approach to Corruption, in A. VON ARNAULD, K. VON DER DECKEN, M. SUSI (eds.), The Cambridge Handbook of New Human Rights. Recognition, Novelty, Rhetoric, Cambridge, 2020, pp. 531-538; K.E. DAVIS, Corruption as a Violation of International Human Rights: A Reply to Anne Peters, in European Journal of International Law, Vol. 29, 2019, pp. 12891296; A. PETERS, Corruption as a Violation of International Human Rights, in European Journal of International Law, Vol. 29, No. 4, 2019, pp. 1251-1287; C. ROSE, The Limitations of a Human Rights Approach to Corruption, in International \& Comparative Law Quarterly, Vol. 65, 2016, pp. 405-438; Z. PEARSON, An International Human Rights Approach to Corruption, in P. LARMOUR, N. WOLANIN (eds.), Corruption and Anti-Corruption, Canberra, 2013, pp. 30-61; J. BACIO TERRACINO, Linking Corruption and Human Rights, in Proceedings of the ASIL Annual Meeting, Vol. 104, 2010, pp. 243-246. $\mathrm{Si}$ veda, inoltre, Segretario Generale del Consiglio d'Europa, Respecting Democracy, Rule of Law and Human Rights in the Framework of the COVID-19 Sanitary Crisis. A Toolkit for Member States, del 7 aprile 2020, SG/Inf(2020)11.

(92) In dottrina, v., supra, nota 37. Si veda, inoltre, Raccomandazione del Comitato dei Ministri del Consiglio d'Europa, on the Protection of Whistleblowers, del 30 aprile 2014, R(2014)7.

(93) GRECO, Corruption Risks and Useful Legal References in the Context of COVID-19, cit., p. 2.

(94) Venti Principi Guida per la Lotta alla Corruzione, supra, nota 14, principio-guida n. 5: "to provide appropriate measures to prevent legal persons being used to shield corruption offences".

(95) Nazioni Unite, Dichiarazione del Segretario Generale sulla corruzione nel contesto del COVID-19, cit. 
(96) Intervento del Ministro della Giustizia italiana, Prof.ssa Marta Cartabia, all' Assemblea Generale delle Nazioni Unite, Sessione Speciale sulla Corruzione, New York, 2-4 giugno 2021.

(97) Ibidem.

(98) Basta guardare ai rapporti valutativi di Paesi quali l’Albania, la Bosnia-Erzegovina, la Macedonia, la Slovacchia, la Slovenia, che sono sintomatici delle dinamiche evolutive dei loro ordinamenti e legislazioni. (99) V., supra, nota 72.

(100) V. TRANSPARENCY INTERNATIONAL, Corruption Perception Index 2020, 2021, pp. 8-10.

(101) Assemblea Generale delle Nazioni Unite, Sessione speciale contro la corruzione, 2-4 giugno 2021. V. Risoluzione 73/191 dell'Assemblea Generale delle Nazioni Unite, Special Session of the General Assembly Against Corruption, del 17 Dicembre 2018, A/RES/73/191.

(102) Assemblea Generale delle Nazioni Unite, Report of the Conference of the States Parties to the United Nations Convention against Corruption on its Preparatory Work for the Special Session of the General Assembly on Challenges and Measures to Prevent and Combat Corruption and Strengthen International Cooperation. Note by the Secretary-General. Addendum, del 17 maggio 2021, A/S-32/2/Add.1.

(103) Per un'analisi più approfondita del contributo delle c.d. soft international organisations nel processo di creazione e di sviluppo del diritto internazionale, v. A. DI STASI, About Soft International Organizations: An Open Question. in R. VIRZO, I. INGRAVALLO (eds.), Evolutions in the Law of International Organizations, Leiden-Boston, 2015, pp. 44-69; A. DI STASI, Il contributo del G20 e del G8 alla definizione di nuove regulae dell'ordine internazionale tra effettività pseudo-istituzionale e partnership di gruppo, in Rivista della cooperazione giuridica internazionale, Vol. 12, 2009, pp. 20-57.

(104) L'Anti-corruption Working Group (ACWG) è stato istituito, in occasione del summit di Toronto del 2010, dai Leaders del G20 per analizzare l'impatto fortemente negativo della corruzione sulla crescita economica, sul commercio e sullo sviluppo. V. G. TARTAGLIA POLCINI, op. cit. 